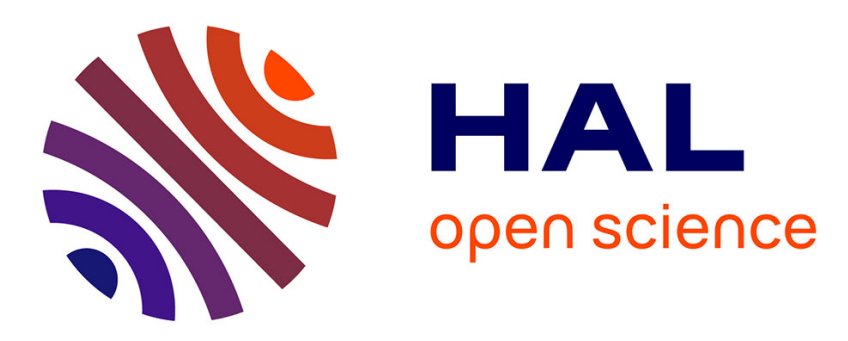

\title{
Les transactions entre la ville et l'inondation pour un urbanisme plus résilient
}

\author{
Sylvain Rode, Mathilde Gralepois, Eric Daniel-Lacombe
}

\section{To cite this version:}

Sylvain Rode, Mathilde Gralepois, Eric Daniel-Lacombe. Les transactions entre la ville et l'inondation pour un urbanisme plus résilient. La Houille Blanche - Revue internationale de l'eau, 2018, 3, pp.34-40. $10.1051 / \mathrm{lhb} / 2018030$. halshs-02284252

\section{HAL Id: halshs-02284252 \\ https://shs.hal.science/halshs-02284252}

Submitted on 11 Sep 2019

HAL is a multi-disciplinary open access archive for the deposit and dissemination of scientific research documents, whether they are published or not. The documents may come from teaching and research institutions in France or abroad, or from public or private research centers.
L'archive ouverte pluridisciplinaire HAL, est destinée au dépôt et à la diffusion de documents scientifiques de niveau recherche, publiés ou non, émanant des établissements d'enseignement et de recherche français ou étrangers, des laboratoires publics ou privés. 


\title{
Les transactions entre la ville et l'inondation pour un urbanisme plus résilient
}

\author{
Sylvain RODE ${ }^{1}$, Mathilde GRALEPOIS ${ }^{2}$ \\ Éric DANIEL-LACOMBE ${ }^{3}$ \\ ${ }^{1}$ Université de Perpignan Via Domitia, Département de Géographie et Aménagement - UMR 5281 ART-Dev - sylvain.rode@univ- \\ perp.fr \\ ${ }^{2}$ Université de Tours, Département Aménagement et Environnement - UMR 7324 CITERES - mathilde.gralepois@univ-tours.fr \\ ${ }^{3}$ École Nationale Supérieure d'Architecture de Paris-la-Villette - edl@edl-architecte.com
}

\begin{abstract}
Les projets d'aménagement urbain en zone inondable se présentent comme des configurations - à la fois spatiales et actorielles - lors desquelles sont mises en ouvre des transactions entre la ville et l'eau afin d'intégrer le risque d'inondation et aboutir à une forme d'urbanisme résilient. L'étude transversale de quatre projets d'aménagement urbain développés en zone inondable constructible en France - le quartier Rives de Maine à Angers, le quartier Berges de la Robine à Narbonne, le quartier de l'Eure St-Nicolas au Havre et le quartier Matra à Romorantin - permet de faire ressortir les grandes catégories de transactions nécessaires à un urbanisme résilient.

Nous identifions tout d'abord trois grands types d'espaces génériques de transaction ville/risque d'inondation, qui sont des solutions d'organisation de l'espace adaptées au risque d'inondation et constituent donc des réponses à l'enjeu de l'urbanisation résiliente des zones inondables : les espaces surélevés, les transparences hydrauliques, l'aménagement paysager de l'interface villeleau. Nous montrons ensuite que la prise en compte de l'inondation dans un projet d'aménagement urbain tient à la capacité des acteurs du projet à articuler registre technique et registre sensible, mais aussi à leur capacité à articuler logique réglementaire et logique de projet. Il s'agit de rendre possible la confrontation des différents acteurs et l'invention de solutions d'organisation de l'espace qui soient le résultat de négociations inventives.
\end{abstract}

Mots-clefs : Inondation - Projet d'aménagement urbain - Transactions - Acteurs - Espace.

\section{Transactions between the city and flood : towards a resilient urban design}

Urban development projects in flood-prone area are presented as configurations - both spatial and actorial - during which transactions between the city and the flood risk are implemented in order to integrate the latter and to achieve a resilient urban design. The cross-cutting study of four urban development projects developed in flood prone built areas in France - the Rives de Maine district in Angers, the Berges de la Robine district in Narbonne, the Eure St-Nicolas district in Le Havre and the Matra district in Romorantin - highlights the broad categories of transactions necessary for a resilient urban design.

We first identify three different kinds of generic spaces allowing transactions between the city and flood: raised spaces, hydraulic transparencies, landscape design of the city/water interface. We then show that the taking into account of flooding in an urban development project is related to the ability of stakeholders to articulate the technical and sensitive registers, as well as a regulatory logic and a project logic. The aim is to make possible the confrontation of the stakeholders and the invention of solutions to organize space which are the result of inventive negotiations.

Key words : Flood - Urban development project - Transactions - Stakeholders - Space.

\section{INTRODUCTION}

Le projet urbain semble désormais envisagé par la littérature scientifique sur l'urbanisme résilient comme « une ouverture du champ des possibles » [Hubert, 2014] potentiellement favorable à une meilleure intégration des risques à la conception urbaine et à l'aménagement. Nous partons du postulat que les projets d'aménagement urbain en zone inondable se présentent comme des configurations (spatiales et actorielles) à l'occasion desquelles doivent être mises en œuvre des transactions nouvelles entre la ville et l'eau afin d'intégrer pleinement le risque d'inondation à la fabrique urbaine et ainsi espérer aboutir à une forme d'urbanisme résilient.

En informatique, la transaction est une suite d'opérations qui fait passer d'un état antérieur à la transaction à un état postérieur. En droit, « la transaction est un contrat par lequel les parties, par des 
concessions réciproques, terminent une contestation née, ou préviennent une contestation à naître » (article $2044 \mathrm{du}$ Code civil). En sciences sociales, la notion de transaction sociale insiste sur l'idée que la décision finale est le résultat d'une transformation des termes de l'échange et de la modification des priorités initiales [Rémy, 1992] des différentes parties pour aboutir à une décision. Le terme de transaction sociale implique que le cadre de la négociation n'est pas seulement technique et matériel, mais exprime également « un jeu de pouvoir et de contre-pouvoir autour d'un problème à résoudre » [Rémy, 2005].

Nous parlons ici de «transactions ville/risque d'inondation » pour définir l'ensemble des modes d'articulation du développement urbain et de la prévention des risques qui doivent être mis en œuvre à l'occasion des projets d'aménagement urbain en zone inondable constructible. Les transactions ville/risque d'inondation se déclinent ainsi en deux registres :

- un registre spatial. Quelles formes particulières d'organisation de l'espace et du bâti sont mises en œuvre aux différentes échelles afin d'adapter les formes architecturales et urbaines à l'inondation dans les projets d'aménagement urbain?

- un registre actoriel. Les transactions ville/risque d'inondation ne se réduisent pas à la mise en œuvre de solutions concrètes et fonctionnelles d'organisation de l'espace urbain. Le projet d'aménagement urbain est aussi un espace-temps où s'opèrent des transactions entre différentes catégories d'acteurs. Or, ces jeux d'acteurs - singuliers au sein du projet d'aménagement urbain en zone inondable [Rode, Gralepois, 2017] - jouent un rôle important dans la réussite ou l'échec de ces transactions ville/risque et, in fine, dans la possibilité d'un urbanisme résilient.

L'objectif de l'article est de mettre en évidence la nécessité, pour espérer parvenir à une forme d'urbanisme résilient, de mettre en œuvre des transactions ville/risque d'inondation qui relient étroitement la gouvernance du projet (rôles et interactions entre les différents acteurs, jeux de négociation) et les solutions d'organisation de l'espace de projet retenues pour intégrer le risque. Car si la littérature opérationnelle et académique sépare souvent les deux aspects, d'une part les solutions spatiales [Gilsoul, 2014] et d'autre part les solutions actorielles [Guevara, Gralepois, 2015], cet article vise à poser les bases d'une réflexion praticienne et académique sur les modalités de convergence de ces deux registres d'action, qui constituent les deux faces indissociables des transactions ville/risque d'inondation pour un urbanisme résilient.

Les résultats présentés dans cet article sont issus du travail d'analyse réalisé sur trois quartiers de villes moyennes projetés récemment en zone inondable (Angers / quartier Rives de Maine, Narbonne / quartier Berges de la Robine et Le Havre / quartier de l'Eure St-Nicolas) dans le cadre du projet de recherche PRÉCIEU ${ }^{1}$. Nous y ajoutons un quatrième cas d'étude, le quartier Matra à Romorantin, développé récemment en zone inondable et inondé en juin 2016, à peine achevé2. Les méthodes employées sont fondées sur la collecte et l'analyse des documents de planification des territoires concernés (Plan de Prévention des Risques d'Inondation - PPRI - et Plan Local d'Urbanisme - PLU) ainsi que des documents d'urbanisme opérationnel à l'échelle du projet d'aménagement urbain (dossiers de création et de réalisation des Zones d'Aménagement Concerté ZAC), une série d'entretiens semi-directifs auprès des acteurs de ces projets (élus, professionnels de l'architecture, de l'urbanisme et de l'aménagement paysager qui interviennent en zone inondable), des visites de terrain, deux comités d'expertise de confrontation des résultats et un séminaire final d'étude.

À travers une analyse transversale de nos quatre cas d'étude, nous identifions trois grands types d'espaces génériques de transaction ville/risque d'inondation : les espaces surélevés, les transparences hydrauliques, les aménagements paysagers (II). L'analyse des transactions spatiales et

\footnotetext{
${ }^{1}$ PRojet d'Étude sur la Contrainte d'Inondation dans les Espaces Urbanisés (PRÉCIEU) financé par le programme Risques, Décision, Territoires (RDT) du Ministère de l'Environnement, du Développement Durable et de l'Énergie (MEDDE). La direction scientifique de ce projet a été assurée par Mathilde Gralepois (Université de Tours) et Sylvain Rode (Université de Perpignan).

${ }^{2}$ Éric Daniel-Lacombe, l'architecte qui a conçu ce quartier, était en effet un membre de l'équipe pluridisciplinaire du projet de recherche PRÉCIEU.
} 
actorielles à l'œuvre dans les projets d'aménagement urbain en zone inondable permet de mettre en exergue un certain nombre de conditions pour intégrer pleinement l'inondation à la fabrique urbaine et ainsi espérer aboutir à une forme d'urbanisme résilient (III).

\section{TROIS GRANDS TYPES D'ESPACES GÉNÉRIQUES DE TRANSACTION VILLE/RISQUE D'INONDATION}

L'analyse de nos cas d'étude permet d'identifier trois grands types d'espaces génériques de transaction ville/risque d'inondation : les espaces surélevés (1), les transparences hydrauliques (2), les aménagements paysagers (3). Déclinés à des échelles différentes, ces types d'espaces de transaction font appel à des expertises professionnelles spécifiques et sont souvent combinés au sein d'un même projet d'aménagement urbain afin d'en assurer la résilience.

\section{II.1 Les espaces surélevés}

La surélévation constitue probablement la réponse la plus classique de l'urbanisme résilient au défi de l'urbanisation en zone inondable. Elle est identifiée aussi bien par la littérature technique [CEPRI, 2015] que par la littérature scientifique [Rode, Gralepois, 2017]. Elle se décline sous plusieurs formes: remblai, bâtiments sur pilotis, bâtiments surélevés sur parkings. Le point commun à ces solutions de surélévation est de porter hors d'eau les espaces fonctionnels sensibles (logements toujours, locaux commerciaux parfois, équipements vulnérables mais aussi voirie ou espaces de circulation). Ce principe de surélévation se retrouve dans les quatre projets étudiés.

La solution du remblai est mise en œuvre à Narbonne, où il s'agit de fabriquer un nouveau sol en rajoutant $50 \mathrm{~cm}$ de terres sur le terrain naturel existant afin de surélever le quartier. Néanmoins ce recours au remblai doit s'accompagner de déblais dans d'autres zones du périmètre de projet afin de compenser le volume remblayé, le principe mis en avant par la réglementation étant de parvenir à un équilibre entre remblai et déblai. La surélévation des bâtiments sur parkings a été mise en œuvre à Angers dans le quartier Saint-Serge : parkings en rez-de-chaussée et dalle surélevée. À Angers toujours, dans le quartier Thiers Boisnet, ce sont les bâtiments et les espaces de circulation piétons qui ont été surélevés. Au Havre, dans le quartier de l'Eure-Saint Nicolas, les bâtiments également sont surélevés, ainsi que les socles des cours à l'échelle de l'îlot. À Romorantin, dans le quartier Matra, la surélévation combine l'usage du remblai et des pilotis, utilisés pour rehausser les immeubles collectifs comme les logements individuels (Fig. 1). Un enjeu important est alors de veiller à ce que les espaces situés sous les constructions sur pilotis demeurent libres et ne soient pas colonisés au bout de quelques années. 

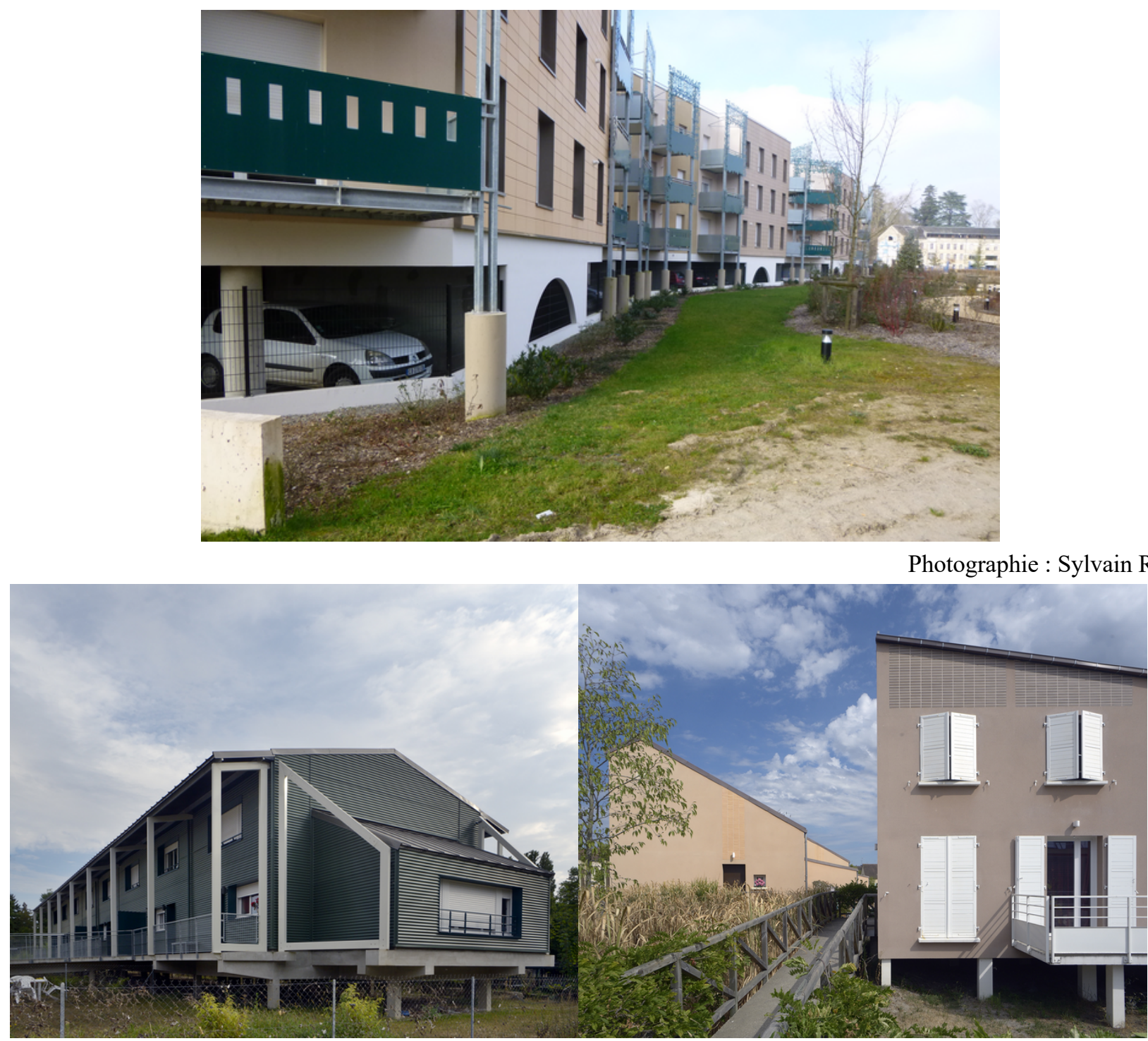

Photographies : Eric Daniel-Lacombe Architecte

Figure 1 : Le quartier Matra à Romorantin : différents types de bâtiments sur pilotis

Si ces réponses architecturales apparaissent vertueuses d'un point de vue de la prévention du risque d'inondation, en contribuant à la résilience des quartiers concernés vis-à-vis de l'inondation, elles posent néanmoins problème en termes de rapport à la rue. De fait, en produisant des formes architecturales et urbaines insulaires, peu lisibles parfois, déconnectées de la rue, ces solutions techniques peuvent fortement contraindre les usages de ces îlots et/ou de ces quartiers, et apparaître ainsi comme étrangères à la ville, comme peu satisfaisantes d'un point de vue du fonctionnement urbain quotidien.

\section{II.2 Les transparences hydrauliques}

Si une première exigence de l'urbanisme résilient est la mise hors d'eau des bâtiments et des espaces de circulation afin de permettre la continuité de la vie urbaine en cas d'inondation, le second enjeu est de faire en sorte que les nouveaux quartiers développés en zone inondable ne fassent pas obstacle au libre écoulement de l'eau de la rivière en crue. Pour cela, les architectesurbanistes qui conçoivent le parti de composition urbaine de ces quartiers doivent y ménager des transparences hydrauliques, c'est-à-dire des espaces libres de constructions qui pourront accueillir l'eau dans le quartier, la guider, la stocker, etc. L'orientation des bâtiments et des voiries surélevées 
doit être pensée en fonction de paramètres hydrauliques. Le travail de composition urbaine se fait ainsi en intégrant des données issues de modélisations hydrauliques. À Romorantin comme à Narbonne, les formes urbaines proposées à l'échelle du quartier mettent en œuvre ce principe des transparences hydrauliques (Fig. 2).

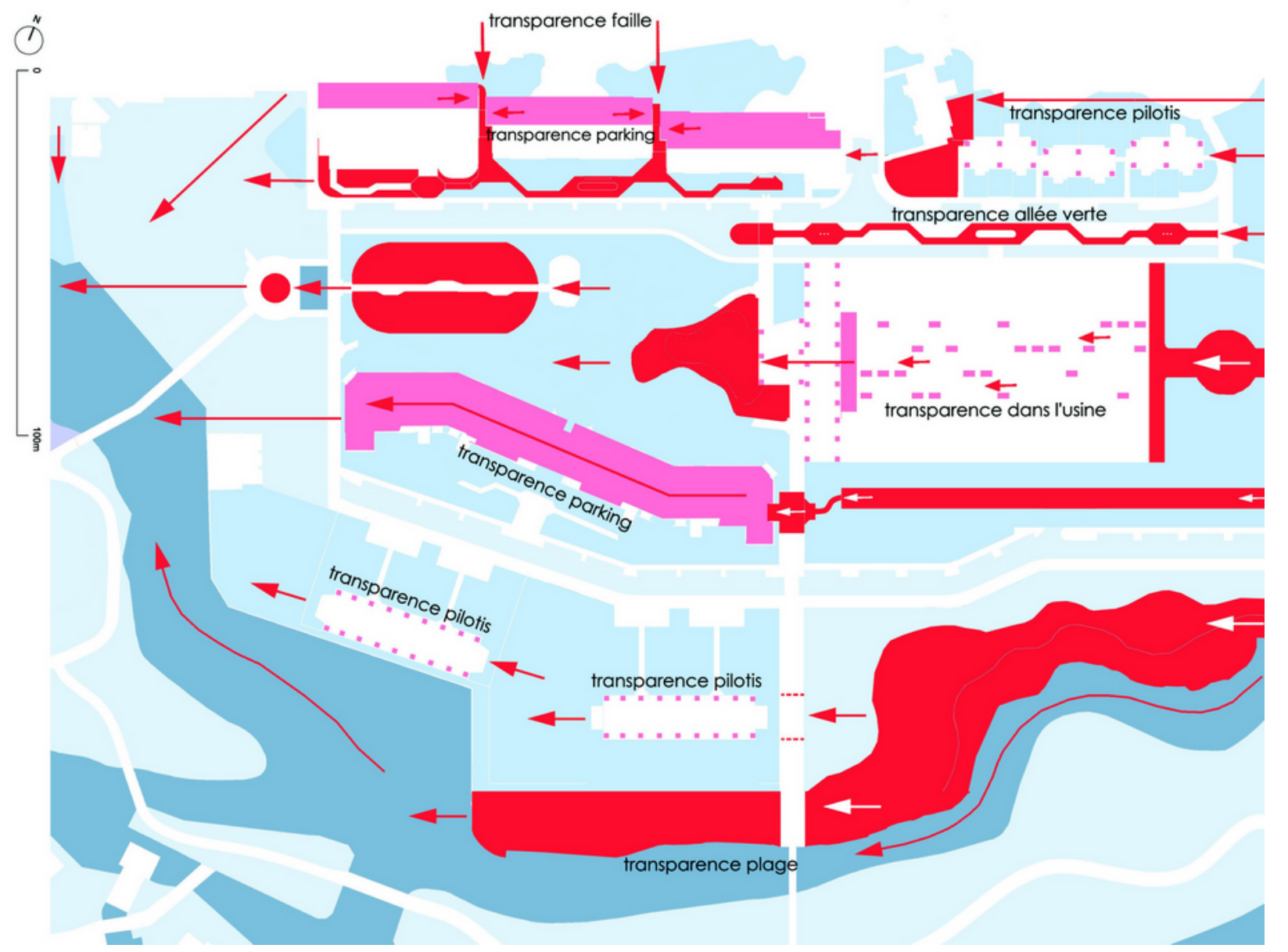

Source : Eric Daniel-Lacombe Architecte

Figure 2 : Le quartier Matra à Romorantin : multiplier les transparences pour permettre la circulation de l'inondation

Là encore, ces espaces de transaction ville/risque d'inondation ne vont pas sans poser question en termes de fabrique de la ville [Gralepois, Rode, 2017]. Comment garantir dans le temps la pérennité des transparences hydrauliques et leur bon fonctionnement, en évitant des occupations illégales, indésirables voire dangereuses (voitures garées dans la trajectoire d'un bassin pluvial par exemple) ? Ces transparences hydrauliques posent également la question de la qualité des espaces publics au cœur des nouveaux quartiers. Leur contribution à l'urbanité des lieux, qui constitue un enjeu majeur, ne va pas de soi. Si ces espaces ne sont envisagés qu'à l'aune de leur seule fonction hydraulique, et non aussi à celle de leur fonction - quotidienne elle - d'espace public, le risque est grand de produire des espaces urbains sans qualités d'usage et peu appropriés par leurs habitants. Les considérations hydrauliques ne peuvent donc pas primer systématiquement sur les considérations urbanistiques, au risque de produire des quartiers certes adaptés à l'inondabilité, mais invivables au quotidien. Elles doivent au contraire être étroitement articulées afin de se combiner positivement et ainsi produire une urbanité résiliente. Un des moyens pour y parvenir est notamment d'apporter un grand soin au traitement paysager de l'interface ville/eau. 


\section{II.3 Les aménagements paysagers}

Lorsqu'il est réduit, comme c'est trop souvent le cas, à des solutions purement techniques fondées sur « une vision très ingénieuriale et technicienne de l'action » [Créton-Cazanave et al., 2016], l'urbanisme résilient peine à produire de la ville et de l'urbanité. Pour pallier cette difficulté, il apparaît nécessaire d'enrichir l'urbanisme résilient d'une dimension sensible, l'enjeu étant alors de signifier et mettre en scène la présence sensible de l'eau dans le quartier. L'urbanisation en zone inondable s'appuie fréquemment sur la valorisation de l'eau visible. Considérée comme une aménité pour les nouveaux quartiers, sa présence - mise en scène par le travail des paysagistes doit contribuer à inverser les images négatives qui souvent caractérisent ces territoires de friches urbaines, industrielles ou portuaires. Cette mise en valeur de la présence sensible de l'eau dans le paysage des nouveaux quartiers, tout en contribuant à leur attractivité et à leur qualité, peut aussi participer, plus ou moins directement, à l'évocation du risque d'inondation. C'est ainsi la possibilité de l'inondation qui doit être signifiée à travers ce travail d'aménagement paysager.

Dans le cadre du projet Angers Rives Nouvelles, l'équipe Grether proposait de traiter l'interface entre la ville et la Maine sous forme de plissés grâce à un système de remblai-déblai et à la création d'un grand parc urbain fluvial. Mise hors d'eau du quartier par rehaussement du terrain naturel et ouverture de la ville sur sa rivière pour affirmer la présence sensible de l'eau étaient donc mobilisées conjointement. Au Havre, l'agence Obras a conçu un «jardin fluvial» pour assurer le traitement paysager de l'interface ville-port, là encore afin d'ouvrir la ville sur ses bassins et affirmer cette présence sensible de l'eau. À Romorantin, l'architecte concepteur du quartier fait appel à un paysagiste reconnu, Bernard Lassus, afin de proposer un grand parc public évoquant de manière sensible cette présence de l'eau au cœur même du quartier Matra (Fig. 3).

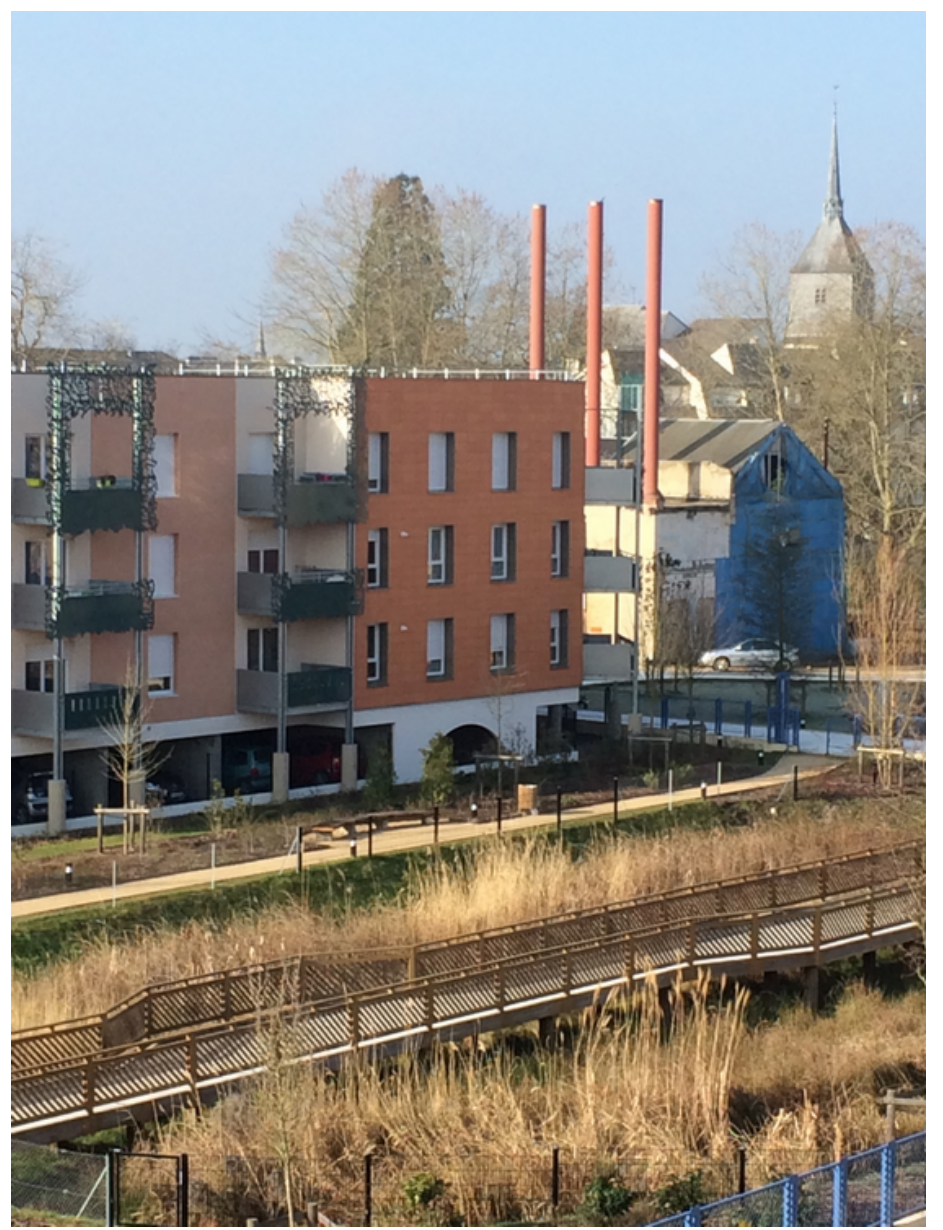

Photographie : Eric Daniel-Lacombe Architecte

Figure 3 : Le quartier Matra à Romorantin : un parc central pour évoquer la présence de l'eau 
Ces trois grands types d'espaces génériques de transactions ville/risque d'inondation ont donc pour but, en les combinant au sein d'un espace de projet, de rendre possible l'urbanisation en zone inondable par la production de formes architecturales et urbaines résilientes à l'inondation. Mais, par delà leur apparente simplicité, ces solutions techniques et fonctionnelles d'organisation de l'espace résultent en réalité de transactions entre les différents acteurs des projets d'aménagement urbain. L'intégration pleine et entière de l'inondation à la fabrique urbaine en faveur d'un urbanisme résilient se joue ainsi largement dans les transactions actorielles singulières à l'œuvre dans le projet d'aménagement urbain en zone inondable.

\section{POUR INTÉGRER PLEINEMENT L'INONDATION À LA FABRIQUE URBAINE}

Dans la droite ligne du paradigme de la transaction, qui se représente « la vie sociale comme étant une confrontation d'une pluralité d'acteurs en relation partiellement conflictuelle et en négociation pour déterminer des zones d'accord en fonction de leur capacité de pression respective » [Rémy, Voyé et Servais, 1978], le projet d'aménagement urbain en zone inondable peut être conçu comme un véritable espace de transactions socio-professionnelles entre acteurs (discussions, échanges, controverses, oppositions, divergences, négociations, compromis, invention, etc.) leur permettant ainsi d'inventer des transactions spatiales (c'est-à-dire qui traduisent dans l'espace urbain la place donnée au risque d'inondation) à la fois techniques et sensibles. Véhiculant « une posture d'action spécifique, celle du dialogue, du partenariat, de la négociation » [Arab, 2004], le projet est censé le permettre. Néanmoins, les rapports de force à l'œuvre entre acteurs, leurs logiques politiques et/ou professionnelles potentiellement discordantes peuvent compliquer les choses.

Les transactions ville/risque d'inondation se jouent alors dans une articulation entre registre technique et registre sensible (1) ainsi que dans une articulation des interventions des différents acteurs du projet (2).

\section{III.1 Articuler registre technique et registre sensible}

Un enjeu majeur des transactions ville/risque d'inondation pour un urbanisme résilient est que les espaces de transaction parviennent à articuler mise hors d'eau des bâtiments et culture sensible du risque en inscrivant la présence sensible de l'eau dans l'espace du projet.

C'est ce qu'exprimait André Bachoc lors du séminaire Risques, Décision, Territoires à mi-parcours qui s'est tenu à Lyon en novembre 2015 en évoquant l'importance de « raconter l'histoire de l'eau » à travers une écriture architecturale, urbaine et paysagère spécifique aux nouveaux quartiers développés en zone inondable. L'enjeu est ainsi de parvenir à rendre l'eau visible sur le territoire, alors même qu'elle n'y est pas présente en permanence. Cette écriture sensible, exprimant les caractéristiques du milieu naturel, doit provoquer des "effets de révélation qui déclenchent la réflexion » (Bachoc, Lyon, 2015). C'est le parti adopté dans le quartier Matra à Romorantin, où « la montée des eaux s'impose à l'imagination » [Daniel-Lacombe, Paquot, 2016] grâce à un ensemble de signes et à un traitement sensible de l'espace du projet. Par exemple, « le bassin de rétention des eaux de pluie dans le parc public est tout autant un catalyseur de l'imaginaire qu'un objet technique. [...] Les présages qu'il porte ne sont pas faciles à interpréter, mais ils attirent l'attention de tous les habitants et font l'objet de conversations et de débats d'opinion à propos du climat, de la rivière, et de ses incidences sur le quartier » [Daniel-Lacombe, Paquot, 2016]. Il s'agit bien ainsi de contribuer à la culture du risque des habitants des quartiers résilients. A contrario, le cas d'Angers montre la nécessité d'aller au-delà d'une simple réponse technique à la prise en compte du risque dans l'aménagement urbain. Certains acteurs des projets Saint-Serge et Thiers Boisnet en ont conscience a posteriori, estimant que la prise en compte des inondations a été "peut-être une réponse exclusivement technique : il faut surélever. Il n'y a pas eu d'approche sensible comme sur Rives Nouvelles, de dire on va prendre en compte la contrainte, mais que cette donnée soit un 
espace de projet. Aujourd'hui on le ferait, mais à leur époque cela n'a pas été fait. On a géré la contrainte de l'eau » (entretien Agence d'Urbanisme de la Région Angevine, 07/04/2014).

De fait, les transactions ville/risque d'inondation ont toutes les chances de ne pas s'avérer pleinement efficaces si elles sont réduites à leur seule dimension technique et réglementaire. En étant une réponse "sectorielle", elles peuvent être efficaces d'un point de vue de la protection contre l'inondation, mais pas du fonctionnement urbain dans son ensemble, de son urbanité. En activant le registre sensible, le travail paysager de mise en visibilité de l'eau aide à développer la compréhension des enjeux liés à la prévention des risques d'inondation.

\section{III.2 Le rôle central du jeu d'acteurs dans les transactions ville/risque d'inondation}

« La notion de projet urbain renvoie aussi à une multiplicité de techniques, parce qu'il se rapporte à plusieurs compétences d'aménagement, de construction, d'écologie. Cette multiplicité de techniques n'a de sens que si elle a une légitimation globale (dans la conception même des choses et dans les moyens d'articuler toutes les techniques) de nature politique. Le projet urbain [...] s'identifie avec un ensemble d'actions inscrites dans la durée et légitimées par le pouvoir politique » [Ingallina, 2001]. Ainsi, pour que les transactions ville/risque d'inondation au sein d'un projet d'aménagement urbain puissent être fertiles, un certain nombre de caractéristiques du jeu d'acteurs doivent également être réunies. Une alchimie entre un maître d'ouvrage et les services de l'État (1) ainsi qu'entre les différents professionnels de l'équipe de maîtrise d'œuvre (2) est tout particulièrement nécessaire à l'invention d'un urbanisme résilient.

\section{III.2.1 La maîtrise d'ouvrage et les services de l'État}

Le rôle des élus locaux, et d'une manière plus générale de la maîtrise d'ouvrage dans ses niveaux régalien et opérationnel [Bernateau, 2001], ne doit pas être sous-estimé. Si ce ne sont pas eux qui dessinent le projet et décident des solutions techniques et sensibles propres à traduire la contrainte hydraulique dans l'aménagement, leur rôle en termes d'élaboration du programme et de conduite du projet n'est pas sans influence sur le cours du projet et ses orientations. Les élus locaux doivent informer le projet en portant une vision pour le territoire, une vision du projet, en fixant un cap et en contribuant à ce qu'il soit tenu tout en faisant preuve de souplesse pour s'adapter aux imprévus. Une certaine continuité politique peut être bienvenue pour éviter le risque de remise en cause des orientations du projet au moment d'alternances politiques. Le cas de Narbonne, où les trois dernières élections municipales ont conduit à des changements de maires et de majorités municipales, illustre de manière patente les conséquences directes qu'une forme d'instabilité politique peut avoir en déstabilisant profondément un projet, en revenant sur les choix d'un prédécesseur, produisant ainsi une forme de paralysie de l'action publique.

Le rôle des services de l'État apparaît essentiel dans un projet d'aménagement urbain en zone inondable. Ils ont en effet pour mission d'élaborer la règle (à travers l'élaboration des Plans de Prévention des Risques d'Inondation) et de s'assurer de son respect. Mais ils doivent aussi faire preuve d'une capacité à intégrer la logique de projet comme un espace-temps propice à une vraie réflexion sur l'intégration du risque d'inondation dans un projet d'aménagement global, c'est-à-dire une opportunité pour inventer des solutions en faveur d'une ville plus résiliente plutôt que comme un simple lieu d'application d'une règle pensée comme intangible. Pour cela, il semble souhaitable que les services de l'État intègrent l'espace de transaction du projet comme un acteur à part entière et non pas qu'ils se considèrent uniquement comme un acteur extérieur, surplombant, celui qui dit la règle. Ils ne peuvent se contenter, comme c'est souvent le cas dans les projets hors zone à risque, d'un rôle «d'observateur vigilant» [Claude, 2006]. En participant pleinement à la dynamique du projet, il devient alors possible pour les services de l'État de se placer dans une logique constructive d'échanges avec les acteurs politiques et professionnels du projet, afin inventer avec eux des solutions d'urbanisme résilient. 
L'espace de transaction ville/risque d'inondation peut alors devenir un lieu de «rencontre institutionnelle [censée être féconde] entre les services de l'État - qui dépassent leur fonction régalienne pour apporter une expertise aiguisée - et les collectivités locales, dont on stimule les capacités d'établir une vision partagée, une vision politique fédératrice » [Bonnet, 2016].

\section{III.2.2 Le rôle des professionnels du projet urbain}

Le rôle et la place des différents professionnels du projet urbain réunis au sein des équipes de maîtrise d'œuvre se trouvent redéfinis dans le projet d'aménagement urbain en zone inondable. Si la «transversalité des métiers» [Bonnet, 2016] est capitale dans les transactions ville/risque d'inondation, il est néanmoins possible d'identifier des enjeux spécifiques selon les métiers.

Les professionnels de la conception urbaine que sont les architectes-urbanistes découvrent souvent la problématique de l'inondation et les enjeux hydrauliques une fois qu'ils sont impliqués dans le projet, c'est du moins le cas dans les premiers projets d'aménagement urbain développés en zone inondable constructible dans la perspective d'inventer un urbanisme résilient. Dans ce cas, ces architectes-urbanistes en charge de la conception du quartier ne sont pas spécialistes de l'urbanisme résilient et ils n'ont donc pas de recette toute faite à mobiliser pour apporter des réponses architecturales et urbaines aux problématiques hydrauliques. L'essentiel n'est-il pas, alors, d'avoir à faire à des professionnels de la conception qui prennent au sérieux la question du risque dans la conception du projet et en fassent un paramètre à part entière de la conception urbaine ? C'est ce qui s'est passé pour le quartier Matra à Romorantin, où l'architecte-urbaniste en charge de la conception du projet a "découvert » la problématique de l'inondation une fois le projet déjà assez avancé. Dans ce cas, les professionnels de la conception urbaine ont accepté de jouer le jeu de l'inondation. Il a fallu apprendre au fur et à mesure de l'avancement du projet, s'acculturer à de nouvelles notions, intégrer de nouveaux paramètres, faire avec la présence incontournable de nouveaux acteurs, mobiliser de nouvelles expertises, et inventer des solutions adaptées au territoire et à son inondabilité, pour finalement en faire un paramètre à part entière de la conception. Ces professionnels de l'urbanisme ont accepté une forme de déstabilisation de leur cadre cognitif et professionnel d'intervention, les obligeant ainsi à délaisser les solutions toutes faites et possiblement reproductibles d'un projet à un autre au profit d'un registre d'invention créative permettant de dégager des réponses propres à un territoire, et adaptées à ses problématiques spécifiques.

Ce type de positionnement de l'architecte-urbaniste intervenant en zone inondable, devant composer avec l'incertitude et inventer des réponses nouvelles et adaptées à l'inondabilité, ne contribue-t-il pas, somme toute, à réaffirmer le caractère prudentiel de sa pratique professionnelle [Champy, 2000], c'est-à-dire la mobilisation de savoirs dans des situations d'incertitude, dans lesquelles on ne peut se contenter d'appliquer un savoir normalisé [Vézinat, 2010] ? Mais dans le même temps, l'intervention des professionnels de la modélisation hydraulique, en cadrant fortement le travail de composition urbaine et en ayant tendance à lui imposer des réponses techniques parfois stéréotypées à la problématique de l'inondabilité, ne limite-t-elle pas ce caractère prudentiel ?

sdans laquelle l'architecte 'décidait' de la forme urbaine d'un quartier » [Ingallina, 2001].

Les paysagistes, «nouveaux venus dans le domaine de l'aménagement de l'espace» [Claude, 2006], jouent eux aussi un rôle majeur dans ces transactions ville/risque d'inondation dans la mesure où leur travail va participer directement à la conception de certaines transactions spatiales, notamment à travers les aménagements paysagers. Présentant la profession de paysagiste concepteur, la Fédération Française du Paysage souligne : « Son savoir-faire n'est pas seulement technique, puisqu'il sait intégrer la dimension subjective, culturelle et sociale du paysage, et mettre en œuvre sa propre créativité ; ni seulement architectural, puisqu'il connaît le végétal, le sol, l'eau, et aussi la complexité, l'évolution et l'incertitude d'un milieu vivant. ${ }^{3}$. De fait, ce rapport subjectif

\footnotetext{
${ }^{3}$ http://www.f-f-p.org/fr/paysagiste-concepteur/definition/
} 
aux éléments naturels et fluctuants du territoire dont se saisit le paysagiste apparaît comme incontournable dans un projet d'aménagement urbain en zone inondable. La mise en scène paysagère, souvent esthétisée, de l'eau conduit à affirmer la présence sensible de l'eau dans la ville, dont on a souligné l'importance afin de ne pas se cantonner à une approche technique de l'urbanisme résilient. Le paysagiste participe donc de la double dimension technique et sensible des transactions ville/risque d'inondation. Toutefois, si son intervention est utile et recherchée, le travail du paysagiste ne suffit pas à résoudre certains problèmes posés par l'urbanisme résilient en termes de production d'urbanité [Rode, Gralepois, 2017] et a finalement "une portée limitée dans le renouvellement des pratiques de l'urbanisme » [Guevara, Gralepois, 2015].

\section{CONCLUSION}

L'invention de transactions ville/risque d'inondation nouvelles et efficaces afin de parvenir à un urbanisme résilient ne doit donc pas se limiter au seul registre technique, au risque de produire des formes architecturales et urbaines stéréotypées, peu satisfaisantes en terme de fonctionnement ordinaire et quotidien. Comme dans tout projet mais peut-être plus encore pour les projets en zone inondable, la capacité des différents acteurs à coopérer pour inventer ensemble des solutions adaptées et situées apparaît déterminante. De fait, la réussite dans la prise en compte de l'inondation par un projet d'aménagement urbain tient largement à la capacité des acteurs de ce projet à créer un espace de transactions qui permette la confrontation des points de vue et contraintes des différents acteurs et l'invention de solutions d'organisation de l'espace qui soient le résultat de négociations inventives et créatives. Lorsque les dispositifs spatiaux retenus comme réponse à la problématique de l'inondabilité ne sont pas le fruit d'une proposition collective des acteurs du projet d'aménagement urbain, et qu'ils ne sont pas appropriés et portés par un collectif d'acteurs, bien souvent ils ne produisent pas la résilience ou l'urbanité recherchée. L'enjeu est aussi de s'assurer du caractère durable de ces solutions d'urbanisme résilient: au-delà de l'invention d'un urbanisme résilient durant la phase de conception urbaine d'un projet de quartier nouveau, il est également nécessaire de faire en sorte que l'urbanisme conserve sa résilience à l'épreuve du temps et de la vie quotidienne des quartiers.

L'enjeu n'est-il pas finalement, pour l'ensemble des acteurs du projet d'aménagement urbain en zone inondable, de parvenir à mieux articuler la logique réglementaire (ce qui est autorisé versus ce qui est interdit) et la logique de projet (l'invention collective de solutions adaptées aux territoires et à leurs enjeux singuliers) ?

\section{RÉFÉRENCES}

Arab N. (2004). - L'activité de projet dans l'aménagement urbain. Processus d'élaboration et modes de pilotage. Le cas de la ligne $B$ du tramway strasbourgeois et d'Odysseum à Montpellier, Thèse de doctorat, Ecole Nationale des Ponts et Chaussées, Aménagement et urbanisme.

Bernateau D. (2001). - «Le transfert de risque entre maître d'ouvrage et maître d'œuvre. Cas de projets complexes ", in Bonnet M., Claude V. et Rubinstein M. (éd.), La commande... de l'architecture à la ville, La Défense : Plan Urbanisme construction architecture, 121-134.

Bonnet F. (2016). - Atout risques, des territoires exposés se réinventent, Marseille, Parenthèses.

CEPRI (Centre Européen pour la Prévention du Risque d'Inondation) (2015). - «Comment saisir les opérations de renouvellement urbain pour réduire la vulnérabilité des territoires inondables face au risque d'inondation ? - Principes techniques d'aménagement ».

Champy F. (2000). - « Les architectes, les urbanistes et les paysagistes », in Paquot T., Lussault M., BodyGendrot S. (dir.), La Ville et l'Urbain, l'état des savoirs, Paris, La Découverte, 215-224.

Claude V. (2006). - Faire la ville. Les métiers de l'urbanisme au XX siècle, Marseille, Parenthèses. 
Créton-Cazanave L., Deroubaix J.-F., Hubert G., Dubois-Maury J., November V. (2016). - «Un « urbanisme résilient» en zone inondable. Le cas des Ardoines, en région Parisienne», in Rudolf F. (dir.), Les villes à la croisée des stratégies globales et locales des enjeux climatiques, Presses de l’Université Laval, 101-131.

Daniel-Lacombe E., Paquot, T. (2016). - «Romorantin : vivre avec l'indiscipline des eaux », EcologiK, $\mathrm{n}^{\circ} 12,38-43$.

Gilsoul N. (2014). - « La ville engloutie», in Terrin J.-J. (dir.), Villes inondables, Prévention, adaptation, résilience, Marseille, Parenthèses, 248-265.

Guevara S., Gralepois M. (2015). - «L'adaptation aux risques d'inondation façonnée par les métiers de la ville », Développement durable et territoires, vol. 6, n³, mis en ligne le 18 décembre 2015, URL: http://developpementdurable.revues.org/11014

Gralepois M., Rode S. (2017). - «L'urbanisme résilient déforme-t-il la ville ?», Risques urbains [En ligne], 17, $\mathrm{n}^{\circ}$ 2, 17 p., URL : https://www.openscience.fr/L-urbanisme-resilient-deforme-t-il-la-ville

Hubert G. (2014). - «Ville et inondation : une cohabitation délicate », in Terrin J.-J. (dir.), Villes inondables. Prévention, adaptation, résilience, Marseille, Parenthèses, 218-233.

Ingallina P. (2001). - Le projet urbain, Paris, PUF, QSJ ?

Rémy J., Voyé L., Servais É. (1978). - Produire ou reproduire? Une sociologie de la vie quotidienne, tome 1, Bruxelles, Les Éditions Vie Ouvrière.

Rémy J. (1992). - «La vie quotidienne et les transactions sociales: perspectives micro ou macrosociologiques », in Blanc M. (dir.) Pour une sociologie de la transaction sociale, Paris, L'Harmattan, 83111.

Rémy J. (2005). - «Négociations et transaction sociale», Négociations, 1, $\mathrm{n}^{0}$ 3, 81-95. DOI : 10.3917/neg.003.0081. URL : https://www.cairn.info/revue-negociations-2005-1-page-81.htm

Rode S., Gralepois M. (2017). - « Towards an Urban Design Adapted to Flood Risk ? », in Vinet F. (ed.), Floods. Volume 2 : Risk Management, ISTE Press, Elsevier, 365-378.

Vézinat N. (2010). - «Une nouvelle étape dans la sociologie des professions en France. Bilan critique autour des ouvrages de Didier Demazière, Charles Gadéa (2009) et Florent Champy (2009) », Sociologie, n³, vol. 1, pp. 413-420, consulté le 28/05/15. En ligne : www.cairn.info/revue-sociologie-2010-3-page413.htm 